\title{
Density matrix numerical renormalization group for non-Abelian symmetries
}

\author{
A. I. Tóth,$^{a, b}$ C. P. Moca, ${ }^{a, c}$ Ö. Legeza ${ }^{a, d}$ and G. Zaránd ${ }^{a}$ \\ a Theoretical Physics Department, Institute of Physics, \\ Budapest University of Technology and Economics, H-1521 Budapest, Hungary \\ ${ }^{b}$ Institute for Theoretische Festkörper Physik, Universität Karlsruhe, D-76128 Karlsruhe, Germany \\ c Department of Physics, University of Oradea, Oradea, Romania \\ ${ }^{d}$ Research Institute for Solid State Physics and Optics, P.O. Box 49, H-1525 Budapest, Hungary
}

(Dated: February 29, 2008)

\begin{abstract}
We generalize the spectral sum rule preserving density matrix numerical renormalization group (DM-NRG) method in such a way that it can make use of an arbitrary number of not necessarily Abelian, local symmetries present in the quantum impurity system. We illustrate the benefits of using non-Abelian symmetries by the example of calculations for the $T$-matrix of the two-channel Kondo model in the presence of magnetic field, for which conventional NRG methods produce large errors and/or take a long run-time.
\end{abstract}

PACS numbers: 71.10.Pm, 71.27.+a, 72.15.Qm, 73.21.La, 75.20.Hr

\section{INTRODUCTION}

Quantum impurity models play a crucial role in our understanding of strongly correlated systems: They appear in the description of correlated mesoscopic structures, 1 they show up in molecular electronics, and many of the properties of correlated bulk systems can also be accounted for using self-consistent quantum impurity models within the dynamical mean field approach ${ }^{2}$ Despite the lot of interest and the large amount of effort invested in understanding these models, we have, unfortunately, very limited tools to describe quantitatively the general properties of a generic quantum impurity model. For some of the quantum impurity models Bethe Ansatz,$\underline{3,4}$ conformal field theory, $\stackrel{5}{=}$ bosonization, $\underline{\underline{6}}$ perturbative calculations, $\stackrel{7,8}{=}$ or a Fermi liquid theory $\underline{\underline{9}}$ can provide a satisfactory explanation. However, these results are usually restricted to some regions in the parameter space. Therefore, even today, the most reliable method to obtain accurate information on a generic quantum impurity model over the whole parameter space and for any frequency is Wilson's numerical renormalization group method (NRG) ${ }^{10}$ originally developed for the onechannel Kondo model (1CKM).

Apart from being extended to compute dynamical properties, $11,12,13,14$ Wilson's method has been used in its original form for a longtime, and it is only recently that this method has been further developed. First, Hofstetter realized that a density matrix NRG (DM-NRG) procedure needs to be introduced in certain cases to avoid spurious results 15 The method of Hofstetter, however, applied the original truncation scheme of Wilson, and therefore has not conserved spectral weights. A remarkable development of the NRG scheme has been the introduction of a complete basis set by Anders et al. 16 which was used to develop the time-dependent DM-NRG algorithm, and it also led to the development of spectral sum-conserving DM-NRG algorithms. 17,18 In the work of Weichselbaum and von Delft, the spectral sum conserving method has been hybridized with a matrix product state approach. 18

Using symmetries is an important element of NRG. For the simplest models, it is usually sufficient to use Abelian symmetries. However, for the more interesting two- and multi-channel models it is crucial to exploit symmetries, since the computational effort needed increases rapidly with the number of electron channels, and it is very important to keep a sufficiently large number of levels in the DM-NRG procedure to achieve good accuracy. Despite the amazing development discussed in the previous paragraph, a general framework in which the advantages of non-Abelian symmetries are exploited in conjunction with DM-NRG was still missing, apart from the generalization of DM-NRG for the case of only one $\mathrm{SU}(2)$ symmetry. 19 The aim of the present paper is to develop such a scheme and to show, how the spectral sum-conserving DM-NRG method can be used in combination with non-Abelian symmetries. For this purpose, we derive a very general recursion formula for the reduced density matrix. We shall show how this formula can be used to evaluate the spectral function of any retarded Green's function of the form

$$
G_{A, B^{\dagger}}^{R}(t) \equiv-i\left\langle[A(t), B(0)]_{\xi}\right\rangle \Theta(t) .
$$

Here $A$ and $B$ are any kind of local fermionic or bosonic operators acting at the impurity site, and $[A, B]_{\xi}=$ $A B-\xi B A$ denotes the commutator $(\xi=1)$ and the anticommutator $(\xi=-1)$ for bosonic or fermionic operators, respectively. In Eq. (1),$\langle\ldots\rangle$ denotes the average with the equilibrium density matrix, $\langle\ldots\rangle=\operatorname{Tr}\{\ldots \varrho\}$. Expressions for static quantities shall also be derived.

Due to the general recursion relation mentioned above, we were able to perform a DM-NRG calculation independently of the type and number of symmetries considered, and to build a completely flexible DM-NRG code that handles symmetries dynamically and "blindly" 21

To demonstrate the benefits of our procedure, we shall present numerical results for the two-channel Kondo model, which is the most basic non-Fermi liquid quantum impurity model,,$\stackrel{20}{,}$ and provides an excellent testing 
ground for multiple non-Abelian symmetries. Furthermore, its study is also motivated by its recent mesoscopic realization in double-dot systems by Potok et al. ${ }^{22}$ As we shall see, conventional NRG techniques and DM-NRG with Abelian symmetries give rather poor results compared to a computation performed using the non-trivial symmetry structure of the model.

The paper is organized as follows. In Section [II we present how one can exploit the internal symmetries of the quantum impurity system in the NRG process. In Section III the general procedure, which permits the use of an arbitrary number of local symmetries, is extended to the spectral sum rule preserving DM-NRG algorithm. In Section IV we provide the general formulas for computing Green's functions in the DM-NRG framework using symmetries. In Section $\mathrm{V}$ we present our numerical results for the local fermions and local composite fermions spectral functions and for the on-shell $T$-matrix in the two-channel Kondo model which strongly support the use of DM-NRG (with the largest possible symmetry of the system) as opposed to the use of NRG. In Section VI we sum up what has been presented. In Appendix VII we give an alternative derivation of the result that the reduced density matrix retains its diagonal form in the course of the reduction for the special case when the symmetry of the system contains only $\mathrm{SU}(2)$ groups from among non-Abelian groups.

\section{THE ROLE OF SYMMETRIES IN THE NRG PROCEDURE}

In the present section we shall briefly discuss, how non-Abelian symmetries appear in the NRG calculations. However, before setting up the general formalism it is useful to discuss the role of non-Abelian symmetries in the specific example of the one-channel Kondo (1CK) model and consider the general case and the general recursion relations only afterwards.

\section{A. Symmetries of the one-channel Kondo model}

In the NRG procedure, Wilson used the following approximation for the $1 \mathrm{CK}$ Hamiltonian ${ }^{10}$

$$
\begin{aligned}
H_{1 C K}=\frac{1}{2} & \mathcal{J} \sum_{\sigma, \sigma^{\prime} \in\{\uparrow, \downarrow\}} \vec{S} f_{0, \sigma}^{\dagger} \vec{\sigma}_{\sigma \sigma^{\prime}} f_{0, \sigma^{\prime}} \\
& +\sum_{n=0}^{\infty} \sum_{\sigma \in\{\uparrow, \downarrow\}} t_{n}\left(f_{n, \sigma}^{\dagger} f_{n+1, \sigma}+\text { h.c. }\right) .
\end{aligned}
$$

In this Hamiltonian $f_{0, \sigma}^{\dagger}$ creates a conduction electron of spin $\sigma$ at the impurity site, and thus the first term describes the interaction between the impurity spin, $\vec{S}$, and this local fermion through an exchange coupling, $\mathcal{J}$. The dynamics of this local fermion is described by a semiinfinite chain, the Wilson chain. Electrons (fermions) move along this semi-infinite chain with an exponentially decreasing hopping amplitude, $t_{n} \propto \Lambda^{-n / 2}$, with $\Lambda$ the discretization parameter 10

The above model has an $\mathrm{SU}_{S}(2)$ symmetry, corresponding to spin rotations, i.e. it is invariant under unitary transformations

$$
H_{1 C K}=\mathcal{U}_{s} H_{1 C K} \mathcal{U}_{s}^{\dagger}
$$

where the unitary operator $\mathcal{U}_{s}$ is generated by the total spin operators,

$$
\begin{aligned}
\mathcal{U}_{s} & \equiv \mathcal{U}_{s}\left(\vec{\omega}_{s}\right)=e^{i \vec{\omega}_{s} \vec{S}_{T}}, \\
\vec{S}_{T} & =\vec{S}+\frac{1}{2} \sum_{n=0}^{\infty} \sum_{\sigma, \sigma^{\prime} \in\{\uparrow, \downarrow\}} f_{n, \sigma}^{\dagger} \vec{\sigma}_{\sigma, \sigma^{\prime}} f_{n, \sigma^{\prime}} .
\end{aligned}
$$

The Hamiltonian $H_{1 C K}$ is also invariant under the action of $\mathrm{SU}_{C}(2)$ rotations in charge space, $\mathcal{U}_{c}=e^{i \vec{\omega}_{c} \vec{C}}$, generated by the operators $C^{x}=\left(C^{+}+C^{-}\right) / 2, C^{y}=$ $\left(C^{+}-C^{-}\right) / 2 i$ and $C^{z}$ with

$$
\begin{aligned}
C^{+} & =\sum_{n=0}^{\infty}(-1)^{n} f_{n, \uparrow}^{\dagger} f_{n, \downarrow}^{\dagger}, \\
C^{z} & =\frac{1}{2} \sum_{n=0}^{\infty} \sum_{\mu=\{\uparrow, \downarrow\}}\left(f_{n, \mu}^{\dagger} f_{n, \mu}-1\right), \\
C^{-} & =\left(C^{+}\right)^{\dagger} .
\end{aligned}
$$

Since the spin symmetry generators commute with the charge symmetry generators, $H_{1 C K}$ possesses a symmetry, $\mathrm{SU}_{S}(2) \times \mathrm{SU}_{C}(2),{ }^{23}$ and consequently, the eigenstates of the Hamiltonian form degenerate multiplets, $\left|i, \underline{Q}_{i}, \underline{Q}_{i}^{z}\right\rangle$, that are classified by their label $i$, the spin and charge quantum numbers: $\underline{Q}_{i} \equiv\left\{S_{i}, C_{i}\right\},{ }^{29}$ and the $z$-components of the charge and spin operators, $\underline{Q}_{i}^{z} \equiv$ $\left\{S_{i}^{z}, C_{i}^{z}\right\}$,

In the following, we shall refer to the quantum numbers $\underline{Q}$ as representation indices, while $\underline{Q}^{z}$ are referred to as $\overline{l a}$ bels of the internal states of a given multiplet. Note that the representation index $\underline{Q}$ defines the dimension of the given irreducible subspace which is $\operatorname{dim}(i)=\operatorname{dim}\left(\underline{Q}_{i}\right)=$ $\left(2 S_{i}+1\right)\left(2 C_{i}+1\right)$ in the example above.

Similar to irreducible subspaces of the Hilbert space, operators can be organized into irreducible tensor operator multiplets. $\stackrel{24}{ }$ One of the simplest examples is provided by the impurity spin, from the components of which we can form the operator triplet as

$$
\left\{A_{m}\right\} \equiv\left\{-\frac{1}{\sqrt{2}} S^{+}, S^{z}, \frac{1}{\sqrt{2}} S^{-}\right\} .
$$

The components of this triplet transform under spin rotations as the eigenstates $|m\rangle$ of $S^{z}$ within a spin $S=1$ multiplet, while they are invariant under charge rotations. This means that $A$ has quantum numbers, $S_{A}=1$ and $C_{A}=0$, and the components of this operator multiplet are labeled by $S_{A}^{z}=m,(m=0, \pm 1)$, while the 
internal charge label is trivially $C_{A}^{z}=0$. Similar to the eigenstates of the Hamiltonian, in our simple example the quantum numbers of an irreducible tensor operator $B$ can be organized into a representation index vector, $\underline{b}=\left(S_{B}, C_{B}\right)$ and its components are labeled by $\underline{b}^{z}=$
$\left(S_{B}^{z}, C_{B}^{z}\right)$ taking the values $S_{B}^{z}=-S_{B},-S_{B}+1, \ldots, S_{B}$ and $C_{B}^{z}=-C_{B},-C_{B}+1, \ldots, C_{B}$. A further example for an $S=1 / 2$ and $C=1 / 2$ operator is formed by the four operators $\left\{f_{0, \uparrow}^{\dagger}, f_{0, \downarrow}^{\dagger},-f_{0, \downarrow}, f_{0, \uparrow}\right\}$.

The Wigner-Eckart theorem ${ }^{30}$ then tells us that, apart from trivial group theoretical factors (Clebsches), the matrix elements of the members of a given operator multiplet and states within two multiplets, $i$ and $j$ are simply related by

$$
\left\langle i, \underline{Q}_{i} \underline{Q}_{i}^{z}\left|B_{\underline{b}_{2}}\right| j, \underline{Q}_{j} \underline{Q}_{j}^{z}\right\rangle=\langle i\|B\| j\rangle\left\langle\underline{Q}_{i} \underline{Q}_{i}^{z} \mid \underline{b}, \underline{b}_{z} ; \underline{Q}_{j} \underline{Q}_{j}^{z}\right\rangle
$$

where $\langle i\|B\| j\rangle$ denotes the reduced (invariant) matrix element of $B$, and the generalized Clebsch-Gordan coefficients are simply defined as

$$
\left\langle\underline{Q}_{i} \underline{Q}_{i}^{z} \mid \underline{b}_{z} \underline{b}_{z} ; \underline{Q}_{j} \underline{Q}_{j}^{z}\right\rangle=\left\langle S_{i} S_{i}^{z} \mid S_{B}, S_{B}^{z} ; S_{j} S_{j}^{z}\right\rangle\left\langle C_{i} C_{i}^{z} \mid C_{B}, C_{B}^{z} ; C_{j} C_{j}^{z}\right\rangle
$$

with the usual $\mathrm{SU}(2)$ Clebsches ${ }^{24}$ on the right hand side. This relation is used extensively in the NRG calculations.

An important property of the unitary transformations above is that they are local in the sense that they decompose into unitary operators which commute with each other and act independently at different sites,

$$
\begin{aligned}
\mathcal{U} & =\mathcal{U}_{s} \times \mathcal{U}_{c} \\
\mathcal{U}_{c} & =\prod_{n} \mathcal{U}_{c, n} \\
\mathcal{U}_{s} & =\prod_{n} \mathcal{U}_{s, n}
\end{aligned}
$$

This decomposition property is crucial for using symmetries in the NRG calculations.

\section{B. General local symmetries on the Wilson chain}

Under rather general conditions, the symmetry considerations above and the NRG procedure can be extended to Hamiltonians of the form

$$
H=\mathcal{H}_{0}+\sum_{n=0}^{\infty}\left(\tau_{n, n+1}+\mathcal{H}_{n+1}\right)
$$

Here $\mathcal{H}_{0}$ contains the interaction between the impurity and the fermionic bath (the site of the impurity is labeled by 0 ), and nearest-neighbors on the Wilson chain are coupled through the hopping terms, $\tau_{n, n+1}$. The $n^{\text {th }}$ onsite term $\mathcal{H}_{n+1}$ describes local correlations/interactions, and it can also account for the absence of the electronhole symmetry.

The usual NRG solves the model Eq. (13) by an iterative diagonalization process. The iteration steps consist of diagonalizing the set of Hamiltonians introduced recursively by

$$
\begin{aligned}
H_{0} & =\mathcal{H}_{0} \\
H_{n+1} & =H_{n}+\tau_{n, n+1}+\mathcal{H}_{n+1} .
\end{aligned}
$$

This recursion is depicted in Fig. 1

Let us now assume that $H$ as well as every $H_{n}$ is invariant under the group $G$, i.e.

$$
\mathcal{U}(g) H_{n} \mathcal{U}^{-1}(g)=H_{n}, \quad n=0,1,2, \ldots,
$$

holds for every $g \in G$, with $\mathcal{U}(g)$ the appropriate unitary operator. Furthermore, let us suppose that $G$ and correspondingly $\mathcal{U}$ can be decomposed into a direct product of $\Gamma$ subgroups $\mathcal{G}_{\gamma}(\gamma=1, \ldots, \Gamma)$, each acting independently on every lattice site,

$$
\begin{aligned}
G & =\mathcal{G}_{1} \times \mathcal{G}_{2} \times \cdots \times \mathcal{G}_{\Gamma}, \\
\mathcal{U}(g) & =\prod_{\gamma=1}^{\Gamma} \mathcal{U}_{\gamma}\left(g_{\gamma}\right)=\prod_{\gamma=1}^{\Gamma} \prod_{n} \mathcal{U}_{\gamma, n}\left(g_{\gamma}\right),
\end{aligned}
$$

Note that in the considerations above the subgroups can be also finite, and $G_{\gamma}$ can represent a crystal field symmetry as well as e.g. the $\mathrm{SU}(3)$ group. However, some of

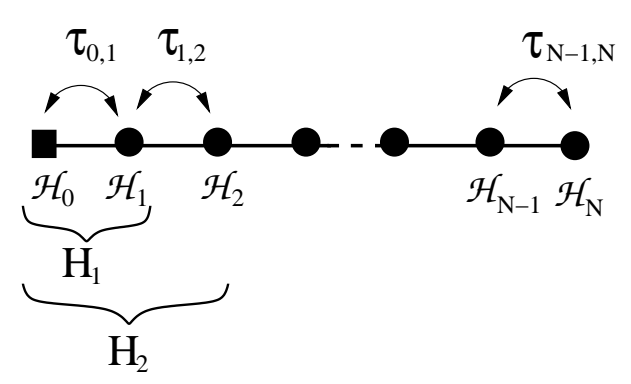

FIG. 1: Construction of the Hamiltonian on the Wilson chain of length $N$. The impurity sits at the square shaped site labeled by 0 , dots represent the further sites. At the zero ${ }^{\text {th }}$ NRG iteration $H_{0}$ is identical with $\mathcal{H}_{0}$. As the $n^{\text {th }}$ site is added to the chain, $H_{n}$ is constructed recursively from the hopping term $\left(\tau_{n, n+1}\right)$, from the on-site energy $\left(\mathcal{H}_{n}\right)$, and from $H_{n-1}$. 
the considerations presented in this paper may not apply for non-compact groups.

The above decomposition is not necessarily unique. Nevertheless, having obtained a specific decomposition of the symmetry, the argument of the previous subsection can be repeated with the only difference that now $\Gamma$ number of quantum numbers classify the irreducible subspaces (multiplets) of the Hamiltonians $H_{n}$,

$$
\underline{Q}=\left\{Q^{1}, Q^{2}, \ldots, Q^{\Gamma}\right\}
$$

and states within the multiplet are then labeled by the internal quantum numbers

$$
\underline{Q}^{z}=\left\{Q^{1, z}, Q^{2, z}, \ldots, Q^{\Gamma, z}\right\}
$$

Similar to the simple $1 \mathrm{CK}$ example, the dimension of a subspace $i$ depends uniquely on its quantum numbers $\underline{Q}_{i}$, i.e. $\operatorname{dim}(i)=\operatorname{dim}\left(\underline{Q}_{i}\right)$.

Operators can also be arranged into irreducible tensor operators, and an irreducible tensor operator multiplet $A$ is correspondingly described by quantum numbers $\underline{a}$, while members of the multiplet are labeled by $\underline{a}^{z}$ with $\underline{a}$ and $\underline{a}^{z}$ being $\Gamma$-component vectors. The Wigner-Eckart theorem, Eq. (8), carries over to the general case too with the slight modification that the Clebsch-Gordan coefficients are now defined as

$$
\begin{aligned}
\left\langle\underline{Q}_{i} \underline{Q}_{i}^{z} \mid \underline{a} \underline{a}^{z} ; \underline{Q}_{j} \underline{Q}_{j}^{z}\right\rangle & \\
& \equiv \prod_{\gamma=1}^{\Gamma}\left\langle Q_{i}^{\gamma} Q_{i}^{\gamma, z} \mid a^{\gamma} a^{\gamma, z} Q_{j}^{\gamma} ; Q_{j}^{\gamma, z}\right\rangle
\end{aligned}
$$

\section{The role of symmetries in the diagonalization procedure}

Before discussing NRG with a complete basis set, $\stackrel{16}{,}$ let us shortly look into how symmetries are used in Wilson's
NRG. In his original work, Wilson constructed approximate eigenstates of $H_{n}$ for a chain of length $n$, iteratively. However, in each iteration the dimension of the Hilbert space increases by a factor $d$, with $d$ the dimension of the local Hilbert space at a single site of the chain with site label $n>0$. Therefore the size of the Hilbert space increases exponentially with $n$, and after a few iterations one must truncate it: Some of the states $i$ are therefore discarded $(i \in D)$, while other states are kept $(i \in K)$, and are used to construct approximate eigenstates for $H_{n+1}$. Symmetries are of great value in this diagonalization procedure: In their presence the $H_{n}$ 's are block diagonal in the representation indices, and the eigenvalue problem can be solved much more efficiently. For some of the physical quantities, it is crucial to increase the number of kept states as much as possible to achieve good numerical accuracy.

In Wilson's original formulation of NRG, symmetries are used in the following way: As discussed above, in the $n^{\text {th }}$ iteration the eigenstates (multiplets) of $H_{n}$ are constructed from the kept multiplets labeled by $u$ with $u \in K$ of the $(n-1)^{t h}$ iteration, which are approximate low-energy eigenstates of $H_{n-1}$, and from a complete set of local states (multiplets), labeled by $\mu$, that live at the $n^{\text {th }}$ site. In the following, we refer to these new approximate eigenstates $i$ as new states, while by analogy with DMRG, we shall call the kept states block states or old states.

In the presence of symmetries, each new multiplet carries representation indices $\underline{Q}_{\tilde{i}}=\left\{Q_{\tilde{i}}^{\gamma}\right\}$, and states within this multiplet are labeled $\underline{b y}^{i}$ the internal quantum numbers $Q_{\tilde{i}}^{z}=\left\{Q_{\tilde{i}}^{\gamma, z}\right\}$. Similarly, local states have quantum numbers $\underline{q}_{\mu}=\left\{q_{\mu}^{\gamma}\right\}$ and are labeled by $\underline{q}_{\mu}^{z}=\left\{q_{\mu}^{\gamma, z}\right\}$. To construct the approximate eigenstates of $H_{n}$, we first use the Clebsches to build new states from the block (old) and local states that transform as irreducible multiplets under the symmetry transformations, $\mathcal{U}(g)$,

$$
\left|\tilde{i}, \underline{Q}_{\tilde{i}}, \underline{Q}_{\tilde{i}}^{z}\right\rangle_{n} \equiv \sum_{\underline{Q}_{u}^{z}, \underline{q}_{\mu}^{z}}\left\langle\underline{q}_{\mu} \underline{q}_{\mu}^{z} ; \underline{Q}_{u} \underline{Q}_{u}^{z} \mid \underline{Q}_{\tilde{i}} \underline{Q}_{\tilde{i}}^{z}\right\rangle\left|u, \underline{Q}_{u}, \underline{Q}_{u}^{z} ; \mu, \underline{q}_{\mu}, \underline{q}_{\mu}^{z}\right\rangle_{n-1}, \quad(u \in \mathrm{K})
$$

These states shall be referred to as the canonical basis from now on. In this basis $H_{n}$ has a block-diagonal structure

$$
\begin{aligned}
& { }_{n}\left\langle\tilde{i}, \underline{Q}_{\tilde{i}}, \underline{Q}_{\tilde{i}}^{z}\left|H_{n}\right| \tilde{j}, \underline{Q}_{\tilde{j}}, \underline{Q}_{\tilde{j}}^{z}\right\rangle_{n} \\
& ={ }_{n}\left\langle\tilde{i}\left\|H_{n}\right\| \tilde{j}\right\rangle_{n} \delta_{\underline{Q}_{\tilde{i}}, \underline{Q}_{\tilde{j}}} \delta_{\underline{Q}_{i}^{z}, \underline{Q}_{j}^{z}},
\end{aligned}
$$

and is diagonalized by a unitary transformation, $\mathcal{O}_{\tilde{i}, i}^{[n]}$

$$
\begin{gathered}
\left|i, \underline{Q}_{i}, \underline{Q}_{i}^{z}\right\rangle_{n}=\sum_{\tilde{i}} \mathcal{O}_{\tilde{i}, i}^{[n]}\left|\tilde{i}, \underline{Q}_{\tilde{i}}, \underline{Q}_{\tilde{i}}^{z}\right\rangle_{n} \underline{\delta}_{\underline{Q}_{i}, \underline{Q}_{\tilde{i}}}{\underline{\delta_{Q}}}_{i}^{z}, \underline{Q}_{\tilde{i}}^{z} \cdot \\
H_{n}\left|i, \underline{Q}_{i}, \underline{Q}_{i}^{z}\right\rangle_{n}=E_{i}^{n}\left|i, \underline{Q}_{i}, \underline{Q}_{i}^{z}\right\rangle_{n}
\end{gathered}
$$

with $E_{i}^{n}$ the eigenenergies of $H_{n}$. Here $\mathcal{O}$ is a blockdiagonal matrix, and its columns in a given symmetry sector are just the eigenvectors of the corresponding sub- 
matrix of ${ }_{n}\left\langle\tilde{i}\left\|H_{n}\right\| \tilde{j}\right\rangle_{n}$ in the canonical basis. In the upcoming iteration, some of these multiplets shall be kept, and form the block states for $(n+1)^{t h}$ iteration, while others are again discarded.

In the iteration step outlined above we need the matrix elements ${ }_{n}\left\langle\tilde{i}\left\|H_{n}\right\| \tilde{j}\right\rangle_{n}$, with $H_{n}=H_{n-1}+\tau_{n-1, n}+$ $\mathcal{H}_{n}$. The matrix elements of $H_{n-1}$ are simply

$$
{ }_{n}\left\langle\tilde{i}\left\|H_{n-1}\right\| \tilde{j}\right\rangle_{n}=E_{u}^{n-1} \delta_{\tilde{i}, \tilde{j}}
$$

where $u$ is the state from which state $\tilde{i}$ has been constructed. Similarly, matrix elements of $\mathcal{H}_{n}$ are given by

$$
{ }_{n}\left\langle\tilde{i}\left\|\mathcal{H}_{n}\right\| \tilde{j}\right\rangle_{n}=\varepsilon_{\mu}^{n} \delta_{\tilde{i}, \tilde{j}}
$$

where $\varepsilon_{\mu}^{n}$ is just the expectation value of $\mathcal{H}_{n}$ with local states within the multiplet $\mu$. Finally, to compute the matrix elements of the hopping $\tau_{n-1, n}$, we use the fact that by symmetry, $\tau_{n-1, n}$ can always be decomposed as

$$
\tau_{n-1, n}=\sum_{\alpha} h_{\alpha}^{[n-1]} \sum_{\underline{c}^{z}}\left[C_{\alpha ; \underline{c}, \underline{c}^{z}}^{[n-1]}\left(C_{\alpha ; \underline{c}, \underline{c}^{z}}^{[n]}\right)^{\dagger}+H . c .\right]
$$

Here $C_{\alpha ; \underline{c}, \underline{c}^{z}}^{[n-1]}$ denotes some creation operator multiplet at site $n-1$ that has quantum numbers $\underline{c}$, and $h_{\alpha}^{[n-1]}$ 's are the hopping amplitudes between sites $n-1$ and $n$. The index $\alpha$ in the equation above labels various " hopping operators". To give a simple example, if we treat the 1 CKM using only $U(1)$ symmetries then one has two hopping operators, $\alpha \in\{1,2\}$, corresponding to $C_{1}^{[n]}=f_{n, \uparrow}^{\dagger}$ and $C_{2}^{[n]}=f_{n, \downarrow}^{\dagger}$. However, if we use the spin $S U(2)$ symmetry, then $\alpha=1$ and $C_{1}^{[n]}=\left\{f_{n, \uparrow}^{\dagger}, f_{n, \uparrow}^{\dagger}\right\}$.

For the reduced matrix elements of $\tau_{n-1, n}$ one obtains using Eq. (21) and the decomposition, Eq. (26), the following formula,

$$
\begin{array}{r}
{ }_{n}\left\langle\tilde{i}\left\|\tau_{n-1, n}\right\| \tilde{j}\right\rangle_{n}=\sum_{\alpha}{ }_{n-1}\left\langle u\left\|C_{\alpha}^{[n-1]}\right\| v\right\rangle_{n-1}\left\langle\nu\left\|C_{\alpha}^{[n]}\right\| \mu\right\rangle^{*} \times \\
D\left(\alpha, \underline{c}, \underline{Q}_{\tilde{i}}, \underline{Q}_{\tilde{j}}, \underline{Q}_{u}, \underline{Q}_{v}, \underline{q}_{\mu}, \underline{q}_{\nu}\right) \delta_{\underline{Q}_{\tilde{i}}, \underline{Q}_{\tilde{j}} \delta_{\underline{Q}_{i}^{z}}, \underline{Q}_{\tilde{j}}^{z}}
\end{array}
$$

Here the state $\tilde{i}$ has been constructed from the state $u$ of the previous iteration and from the local state $\mu$, while $\tilde{j}$ has been constructed from $v$ and $\nu$. The functions

$D\left(\alpha, \underline{c}, \underline{Q}_{\tilde{i}}, \underline{Q}_{\tilde{j}}, \underline{Q}_{u}, \underline{Q}_{v}, \underline{q}_{\mu}, \underline{q}_{\nu}\right)$ denote group theoretical factors,

$$
\begin{aligned}
& D\left(\alpha, \underline{c}, \underline{Q}_{\tilde{i}}, \underline{Q}_{\tilde{j}}, \underline{Q}_{u}, \underline{Q}_{v}, \underline{q}_{\mu}, \underline{q}_{\nu}\right)=\operatorname{sgn}(C, \mu) \sum_{\underline{C}^{z}} \sum_{\underline{Q}_{u}^{z}, \underline{Q}_{v}^{z}} \sum_{\underline{q}_{\mu}^{z}, \underline{q}_{\nu}^{z}}\left\langle\underline{Q}_{\tilde{i}} \underline{Q}_{\tilde{i}}^{z} \mid \underline{q}_{\mu} \underline{q}_{\mu}^{z} ; \underline{Q}_{u} \underline{Q}_{u}^{z}\right\rangle\left\langle\underline{Q}_{\tilde{i}} \underline{Q}_{\tilde{i}}^{z} \mid \underline{q}_{\nu} \underline{q}_{\nu}^{z} ; \underline{Q}_{v} \underline{Q}_{v}^{z}\right\rangle{ }^{*} \times \\
& \left\langle\underline{Q}_{u} \underline{Q}_{u}^{z} \mid \underline{c} \underline{c}^{z} ; \underline{Q}_{v} \underline{Q}_{v}^{z}\right\rangle\left\langle\underline{q}_{\nu} \underline{q}_{\nu}^{z} \mid \underline{c} \underline{c}^{z} ; \underline{q}_{\mu} \underline{q}_{\mu}^{z}\right\rangle^{*}
\end{aligned}
$$

where the $\operatorname{sign}$ function $\operatorname{sgn}(C, \mu)= \pm 1$ arises as one commutes the creation operators implicitly present in the local state $\mu$ over the operator $C^{[n-1]}$, and it is negative if $C_{\alpha}^{[n]}$ is a fermionic operator and the local state $\mu$ is constructed from an odd number of fermions, otherwise it is positive. The local matrix elements, $\left\langle\mu\left\|C_{\alpha}^{[n]}\right\| \nu\right\rangle$ are the same for all sites and can easily be determined, while ${ }_{n-1}\left\langle u\left\|C^{[n-1]}\right\| v\right\rangle_{n-1}$ can be computed from the previous iteration by recursion. In fact, for any operator $A$, acting on sites $m<n$, and whose matrix elements are known in the iteration $n-1$, we have the following recursion relation

$$
{ }_{n}\langle\tilde{i}\|A\| \tilde{j}\rangle_{n}={ }_{n-1}\langle u\|A\| v\rangle_{n-1} F\left(\underline{a}, \underline{Q}_{\tilde{i}}, \underline{Q}_{\tilde{j}}, \underline{Q}_{u}, \underline{Q}_{v}, \underline{q}_{\mu}\right) \delta_{\underline{q}_{\mu}, \underline{q}},
$$


where the factor $F\left(\underline{a}, \underline{Q}_{\tilde{i}}, \underline{Q}_{\tilde{j}}, \underline{Q}_{u}, \underline{Q}_{v}, \underline{q}_{\mu}\right)$ is given by the following expression

$$
\begin{aligned}
F\left(\underline{a}, \underline{Q}_{\tilde{i}}, \underline{Q}_{\tilde{j}}, \underline{Q}_{u}, \underline{Q}_{v}, \underline{q}_{\mu}\right)= & \operatorname{sgn}(A, \mu) \frac{1}{\operatorname{dim}(a) \operatorname{dim}(\tilde{j})} \sum_{\underline{Q}_{\tilde{j}}^{z}, \underline{a}^{z}}\left\langle\underline{Q}_{\tilde{i}} \underline{Q}_{\tilde{i}}^{z} \mid \underline{a}_{\underline{a}}^{z} ; \underline{Q}_{\tilde{j}} \underline{Q}_{\tilde{j}}^{z}\right\rangle^{*} \times \\
& \sum_{\underline{Q}_{u}^{z}, \underline{Q}_{v}^{z}} \sum_{\underline{q}_{\mu}^{z}}\left\langle\underline{Q}_{\tilde{i}} \underline{Q}_{\tilde{i}}^{z} \mid \underline{q}_{\mu} \underline{q}_{\mu}^{z} ; \underline{Q}_{u} \underline{Q}_{u}^{z}\right\rangle\left\langle\underline{Q}_{\tilde{j}} \underline{Q}_{\tilde{j}}^{z} \mid \underline{q}_{\mu} \underline{q}_{\mu}^{z} ; \underline{Q}_{v} \underline{Q}_{v}^{z}\right\rangle^{*}\left\langle\underline{Q}_{u} \underline{Q}_{u}^{z} \mid \underline{a}^{z} ; \underline{Q}_{v} \underline{Q}_{v}^{z}\right\rangle
\end{aligned}
$$

One drawback of the algorithm above is that the eigenstates of $H_{n}$ constructed this way do not form a complete basis set on the Wilson chain of length $n$, since states descendant from the discarded states of the previous iteration are missing. However, as it was recently shown, $\underline{16}$ one can construct a complete basis of the Wilson chain in a slightly different way: Let us consider a chain of length $N$, and construct approximate eigenstates of $H_{n}$ that, however, live at all sites of this chain,

$$
\left|i, \underline{Q}_{i}, \underline{Q}_{i}^{z}\right\rangle_{n} \rightarrow\left|i, \underline{Q}_{i}, \underline{Q}_{i}^{z} ; e\right\rangle_{n} .
$$

In this equation $e$ just labels the $d^{N-n}$ independent 'environment' states living at the last $N-n$ sites of the chain. The internal structure of these environment states is not important, only their degeneracy shall play some role. The previous iterative construction carries over to these states, too. By construction, discarded states (together with their environment state) form a complete basis set:

$$
\mathbb{1}=\sum_{n=0}^{N} \sum_{i \in \mathrm{D}} \sum_{e} \sum_{\underline{Q}_{i}^{z}}\left|i, \underline{Q}_{i}, \underline{Q}_{i}^{z} ; e\right\rangle_{n n}\left\langle i, \underline{Q}_{i}, \underline{Q}_{i}^{z} ; e\right|,
$$

where $i \in D$ refers to the fact that only discarded states appear in the sum. In Eq. (32) all states are considered discarded in the last iteration, $n=N$. We remark that, in reality, the summation in the expression above starts only at a value $n=M$, where the first truncation is carried out. Fig. 2 illustrates the structure of this complete basis. In the formulation of the DM-NRG algorithm we shall use several times the completeness relation, Eq. (32).

\section{CONSTRUCTION OF THE REDUCED DENSITY MATRIX USING SYMMETRIES}

In the DM-NRG procedure on a Wilson chain of length $N$, the equilibrium density matrix is approximated by

$$
\begin{aligned}
\varrho & =\sum_{n=0}^{N} \varrho^{[n]} \\
\varrho^{[n]} & =\sum_{\underline{Q}_{i}^{z}, i, e} \frac{e^{-\beta E_{i}^{n}}}{\mathcal{Z}}\left|i, \underline{Q}_{i}, \underline{Q}_{i}^{z} ; e\right\rangle_{n n}\left\langle e ; i, \underline{Q}_{i}, \underline{Q}_{i}^{z}\right|,
\end{aligned}
$$

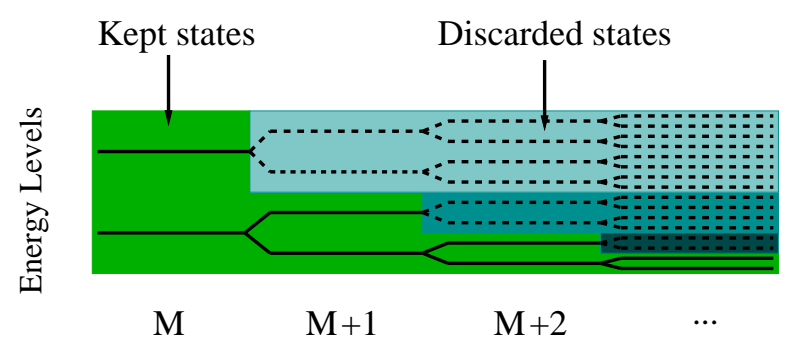

FIG. 2: (color online) A complete basis of a Wilson chain represented as the exponentially increasing number of energy levels belonging to the successive iterations. Continuous/dashed lines represent kept, low-energy/discarded, high-energy levels, respectively. For the consecutive iteration steps the distances between the levels illustrates how the energy resolution of NRG gets exponentially refined.

with $\beta=1 / k_{B} T$ the Boltzmann factor and

$$
\mathcal{Z}=\sum_{n=0}^{N} \sum_{\underline{Q}_{i}^{z}, i} e^{-\beta E_{i}^{n}} d^{N-n}
$$

the partition function. In Eq. (35) the factor $d^{N-n}$ accounts for the degeneracy of the environment states in iteration $n$, i.e. for the local degrees of freedom at sites $m>n$. Since the eigenenergies do not depend on the internal quantum numbers, the expression for the partition function can be simplified to

$$
\begin{array}{r}
\mathcal{Z}=\sum_{n=0}^{N} \sum_{i} \operatorname{dim}(i) e^{-\beta E_{i}^{n}} d^{N-n}, \\
\operatorname{dim}(i) \equiv \prod_{\gamma=1}^{\Gamma} \operatorname{dim}\left(Q_{i}^{\gamma}\right),
\end{array}
$$

with $\operatorname{dim}\left(Q_{i}^{\gamma}\right)$ being the dimension of an irreducible subspace having the representation index $Q_{i}^{\gamma}$.

The concept of the reduced density matrix 15 arises naturally as one starts to calculate Green's functions with NRG. More precisely, the quantity that shows up in the calculations is the truncated reduced density matrix, defined as

$$
R^{[n]}=\operatorname{Tr}_{\left\{e_{n}\right\}}\left\{\sum_{m>n} \varrho^{[m]}\right\},
$$


where one traces over environment states $e_{n}$ at sites $m>$ $n$. This truncated reduced density matrix clearly satisfies the recursion relations

$$
\begin{aligned}
R^{[N]} & =\varrho^{[N]} \\
R^{[n-1]} & =\sum_{i \in D} \frac{e^{-\beta E_{i}^{n-1}} d^{N-n+1}}{\mathcal{Z}}\left|i, \underline{Q}_{i}, \underline{Q}_{i}^{z}\right\rangle_{n n}\left\langle i, \underline{Q}_{i}, \underline{Q}_{i}^{z}\right| \\
& +\operatorname{Tr}_{\text {site } n}\left\{R^{[n]}\right\} .
\end{aligned}
$$

Note that the environment variable is missing in the first term of the second expression since it has been traced over. The first term accounts for the contribution of discarded states, while the second term has matrix elements between the kept states only.

To construct the matrix elements of $R^{[n]}$ we first show by induction that $R^{[n]}$ is scalar under symmetry operations. This is clearly true for the first term in Eq. (39). To show that the second term is also invariant, we simply need to use the locality property of the symmetry transformations, i.e. that on the first $n$ sites $U(g) \equiv L(g) V(g)$, where $L(g)$ transforms the local states on site $n$, while $V(g)$ transforms states at sites $0 \leq m \leq n-1$, and $L$ and $V$ commute with each other. Therefore, because of the trace of operators being invariant under cyclic permutations, we have

$$
\begin{aligned}
\operatorname{Tr} & \left\{L V R^{[n]} V^{+} L^{+}\right\}=\operatorname{Tr}_{\text {site } n}\left\{V R^{[n]} V^{+}\right\} \\
= & V \operatorname{Tr}_{\text {site } n}\left\{R^{[n]}\right\} V^{+}=U \operatorname{Tr}_{\text {site } n}\left\{R^{[n]}\right\} U^{+} .
\end{aligned}
$$

However, since $U R^{[n]} U^{+}=R^{[n]}$ by assumption, we immediately obtain that

$$
\operatorname{Tr}_{\text {site } n}\left\{R^{[n]}\right\}=U \operatorname{Tr}_{\text {site } n}\left\{R^{[n]}\right\} U^{+},
$$

implying that

$$
U R^{[n-1]} U^{+}=R^{[n-1]}
$$

for $R^{[n-1]}$, too. This equation means that $R^{[n]}$ is scalar and therefore, by the Wigner-Eckart theorem we have

$$
\begin{aligned}
{ }_{n}\left\langle i, \underline{Q}_{i}, \underline{Q}_{i}^{z}\left|R^{[n]}\right| j, \underline{Q}_{j}, \underline{Q}_{j}^{z}\right\rangle_{n} & \\
& ={ }_{n}\left\langle i\left\|R^{[n]}\right\| j\right\rangle_{n} \underline{\delta}_{i}, \underline{Q}_{j} \delta_{\underline{Q}_{i}^{z}, \underline{Q}_{j}^{z}}
\end{aligned}
$$

The matrix elements of $\left\langle i\left\|R^{[n]}\right\| j\right\rangle$ between discarded states simply derive from the first term in Eq. (39). To perform the trace in Eq. (39) and to construct the explicit relation between the kept matrix elements of $R^{[n-1]}$ and $R^{[n]}$ some more work is needed. First, we rotate $R^{[n]}$ to the canonical basis

$$
{ }_{n}\left\langle\tilde{i}\left\|\tilde{R}^{[n]}\right\| \tilde{j}\right\rangle_{n}=\mathcal{O}_{\tilde{i}, i}^{[n]}{ }_{n}\left\langle i\left\|R^{[n]}\right\| j\right\rangle_{n}\left(\mathcal{O}^{-1}\right)_{j \tilde{j}}^{[n]}
$$

Then, using the fact that $\operatorname{Tr}_{\text {site } n}\left\{R^{[n]}\right\}$ is diagonal in the symmetry quantum numbers and labels, we can trace over the local states at site $n$ using the recursion relation Eq. (21) to obtain the matrix elements between the kept states $u, v \in K$ as

$$
\begin{aligned}
{ }_{n-1}\langle u & \left.\left\|R^{[n-1]}\right\| v\right\rangle_{n-1} \\
& =\sum_{\sum_{i, \tilde{j}, \underline{q}_{\mu}, \mu}} \frac{\operatorname{dim}(\tilde{i})}{\operatorname{dim}(u)}\left\langle\tilde{i}\left\|\tilde{R}^{[n]}\right\| \tilde{j}\right\rangle_{n} \delta_{\underline{Q}_{\tilde{i}}, \underline{Q}_{\tilde{j}}} .
\end{aligned}
$$

Here the tilde over the sum indicates that in the summation over $\tilde{i}$ and $\tilde{j}$ only those states are considered which have been constructed from $u(\tilde{i} \Leftarrow u)$ and $v(\tilde{j} \Leftarrow v)$ in Eq. (21), respectively. This is a very powerful expression, which applies to essentially any type of symmetry.

\section{SPECTRAL FUNCTION COMPUTATION}

The quantity that describes the linear response of a static system to a time-dependent perturbation and which is to be calculated in the DM-NRG framework is the retarded Green's function. For two irreducible tensor operators it is defined as:

$$
G_{A_{\underline{a}, \underline{a} z}, B_{\underline{b}, \underline{b} z}^{\dagger}}^{\dagger}(t)=-i\left\langle\left[A_{\underline{a}, \underline{a}_{z}}(t), B_{\underline{b}_{\underline{b}},}^{\dagger}(0)\right]\right\rangle \Theta(t) .
$$

By symmetry, however, this Green's function is non-zero only if the spectral operators $A$ and $B$ transform accordingly to the same representation i.e.

$$
G_{A_{\underline{a}, \underline{a}_{z}, B_{\underline{b}, \underline{b}}}^{\dagger}}^{R}(t)=G_{A, B^{\dagger}}^{R}(t) \delta_{\underline{a}, \underline{b}} \delta_{\underline{a}_{z}, \underline{b}_{z}},
$$

and it is independent of the value of $\underline{a}_{z}=\underline{b}_{z}$. Note that in this expression the representation index $\underline{b}$ and its labels $\underline{b}_{z}$ are the quantum numbers that characterize the operator $B$ and not $B^{\dagger}$.

In the reduced density matrix formalism we can generalize the procedure outlined in Ref. [16] even in the presence of non-Abelian symmetries to obtain the following form for the Laplace transform of the Green's function 


$$
\begin{aligned}
G_{A, B^{\dagger}}^{R}(z)=\sum_{n=0}^{N} & \sum_{i \in D, K} \sum_{(j, k) \notin(\mathrm{K}, \mathrm{K})}\left\langle i\left\|R^{[n]}\right\| j\right\rangle_{n} \\
& \times\left[\frac{{ }_{n}\left\langle k\left\|A^{\dagger}\right\| j\right\rangle_{n n}^{*}\left\langle k\left\|B^{\dagger}\right\| i\right\rangle_{n}}{z+\frac{1}{2}\left(E_{i}^{n}+E_{j}^{n}\right)-E_{k}^{n}} \frac{\operatorname{dim}(k)}{\operatorname{dim}(a)}-\xi \frac{{ }_{n}\left\langle j\left\|B^{\dagger}\right\| k\right\rangle_{n n}\left\langle i\left\|A^{\dagger}\right\| k\right\rangle_{n}^{*}}{z-\frac{1}{2}\left(E_{i}^{n}+E_{j}^{n}\right)-E_{k}^{n}} \frac{\operatorname{dim}(i)}{\operatorname{dim}(a)}\right],
\end{aligned}
$$

Remarkably, this formula contains exclusively the reduced matrix elements and the dimensions of the various multiplets. Here the second sum is over all the multiplets $i, j, k$ of the given iteration subject to the restriction that $j, k$ do not belong to kept states at the same time and no summation is needed for states within the multiplets. In Eq. (48) $\operatorname{dim}(a)=\prod_{\gamma=1}^{\Gamma} \operatorname{dim}\left(a^{\gamma}\right)$ is the dimension of the operator multiplet $A_{\underline{a}, \underline{a}_{z}}$. We note that the irreducible matrix elements of $R^{[n]}$ are identical with the original ones since $R^{[n]}$ is invariant under all symmetry transformations, i.e. it is a rank 0 object with respect to all symmetries. Eq. (48) explicitely shows that unless $A$ and $B$ have the same quantum numbers ( $\underline{\mathrm{a}}=\underline{\mathrm{b}}), G_{A, B^{\dagger}}^{R}(z)=0$.

\section{NUMERICAL RESULTS}

In this section we show the advantages of using DMNRG as opposed to NRG, and illustrate the benefits of using non-Abelian symmetries by applying DM-NRG to the two-channel Kondo (2CK) model. This model is exciting in itself as it possesses a non-Fermi liquid type of fixed point and it provides the simplest descriptions of the double dot system used recently to realize the $2 \mathrm{CK}$ state ${ }^{22}$ This 2 CK state is very fragile and in a magnetic field the difference between the NRG and DM-NRG results are substantial.

In the Wilson approach, the 2CKM is described by the following Hamiltonian,

$$
\begin{gathered}
H_{2 C K}=\frac{1}{2} \vec{S} \sum_{\alpha \in\{1,2\}} \mathcal{J}_{\alpha} \sum_{\sigma, \sigma^{\prime} \in\{\uparrow, \downarrow\}} f_{0, \alpha, \sigma}^{\dagger} \vec{\sigma}_{\sigma \sigma^{\prime}} f_{0, \alpha, \sigma^{\prime}}+ \\
+\sum_{n=0}^{\infty} \sum_{\alpha \in\{1,2\}} \sum_{\sigma \in\{\uparrow, \downarrow\}} t_{n}\left(f_{n, \alpha, \sigma}^{\dagger} f_{n, \alpha, \sigma}+\text { h.c. }\right)
\end{gathered}
$$

where we have introduced $\alpha \in\{1,2\}$ for labeling the two types of electrons. This additional channel label is the only difference compared to the one-channel Kondo Hamiltonian (cf. Eq. (2)).

In this model, the number of carriers is conserved in both channels corresponding to a $\mathrm{U}_{C 1}(1) \times \mathrm{U}_{C 2}(1)$ symmetry. However, due to the presence of electron-hole symmetry, these charge symmetries are augmented to $\mathrm{SU}(2)$ symmetries, and the Hamiltonian above is also invariant under $\mathrm{SU}_{S}(2) \times \mathrm{SU}_{C 1}(2) \times \mathrm{SU}_{C 2}(2)$ transformations. On the other hand, one can solve this Hamilto- nian using exclusively $\mathrm{U}(1)$ symmetries, $\mathrm{U}_{S}(1) \times \mathrm{U}_{C 1}(1) \times$ $\mathrm{U}_{C 2}(1)$. This model is thus ideal for testing our flexible methods.

If a local magnetic field is coupled to the impurity spin through a term $g \mu_{B} B S^{z}$, then from among the total spin generators (Eq. (5)) solely $S_{T}^{z}$ will commute with the Hamiltonian. That is, the spin $\mathrm{SU}(2)$ symmetry of the system reduces to $\mathrm{U}(1)$. Therefore, in a magnetic field we can either use the symmetry $\mathrm{U}_{S}(1) \times \mathrm{SU}_{C 1}(2) \times \mathrm{SU}_{C 2}(2)$ for our calculations, or restrict ourself to U(1) symmetries only: $\mathrm{U}_{S}(1) \times \mathrm{U}_{C 1}(2) \times \mathrm{U}_{C 2}(2)$.

As a test, we computed the retarded Green's function, $G_{f_{0, \alpha, \uparrow}, f_{0, \alpha, \uparrow}^{\dagger}}^{R}(\omega)$ and the corresponding spectral function $\rho_{f_{0, \alpha, \uparrow}}(\omega)$ both in the presence and in the absence of magnetic field. All numerical results presented were obtained at zero temperature, and the dimensionless couplings were $\mathcal{J}_{\alpha}=0.2$ for both channels and all runs. The discretization parameter $\Lambda=2$ was used in all cases, and for each symmetry combinations we have retained a maximum number of 1350 multiplets in each iteration.

In Fig. 3 we show data for the local fermion's spectral function in the absence of magnetic field obtained through the NRG and the DM-NRG approaches using the two symmetry groups mentioned above. The Kondo scale $T_{K}$ in Fig. 3 is the scale at which the $2 \mathrm{CK}$ state forms, and it is defined as the frequency where the $T$ matrix of the $2 \mathrm{CK}$ model drops to half of its value assumed at $\omega=0$ at the 2 CK fixed point $\underline{25}$

The first important test is the fulfillment of the spectral sum rules. These are always satisfied in the DMNRG calculations independently of the symmetry group used, whereas the NRG data violate the sum rule to over $15 \%$ if the number of kept multiplets is 1350 corresponding to $\approx 7 \times 10^{4}$ states. Fig. 3. (b) shows that the expected $\sqrt{\omega}$ behavior around the 2CK fixed point is nicely recovered by both methods, but a sufficiently large number of multiplets must be kept in the DM-NRG approach, meaning that in this case the larger symmetry group must be used. Fig. 3. (c) demonstrates that, in spite of fulfilling the spectral sum rules still the DMNRG data do not show the expected asymptotics for low frequencies if the number of multiplets kept is not sufficient. It is quite remarkable that only the DM-NRG procedure using non-Abelian symmetries was able to get close to the exact value of the spectral function at $\omega=0$, $\rho_{f_{0, \alpha, \uparrow}}(\omega=0)=0.25$. 


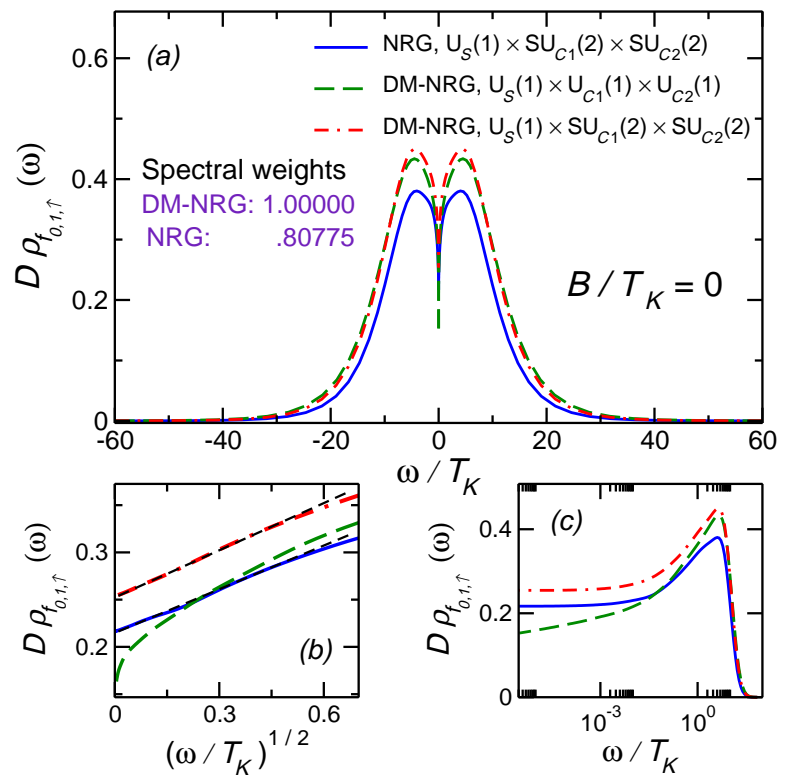

FIG. 3: (color online) Dimensionless spectral function of $f_{0,1, \uparrow}$ normalized by $D$, the bandwidth cut-off, as a function of $\omega / T_{K}$ in the absence of magnetic field obtained with DMNRG and with NRG using the symmetries: $\mathrm{U}_{S}(1) \times \mathrm{SU}_{C 1}(2) \times$ $\mathrm{SU}_{C 2}(2)$ and $\mathrm{U}_{S}(1) \times \mathrm{U}_{C 1}(1) \times \mathrm{U}_{C 2}(1)$. (a) Comparison between the spectral weights of the DM-NRG and NRG results: DM-NRG fulfills the sum rule entirely even when the used symmetry group and therefore the number of kept states is largely reduced. NRG violates the sum rule to over $15 \%$ if the number of kept states is $\approx 7 \times 10^{4}$ in each iteration. (b) The same spectral functions as a function of $\sqrt{\omega / T_{K}}$. If a sufficient number of states is kept, i.e. when using larger symmetry groups, the expected $\sqrt{\omega}$ behavior around the 2CK fixed point is nicely recovered. (c) The same spectral functions on a logarithmic scale.

The presence of magnetic field generates a new scale, $\stackrel{3}{=}$

$$
T_{h} \equiv C_{h} \frac{B^{2}}{T_{K}},
$$

where we have fixed the somewhat arbitrary constant to $C_{h} \approx 60 \underline{26}$ This scale is usually referred to as the renormalized magnetic field acting on the impurity, and below this scale the non-Fermi liquid physics is destroyed.

In the presence of magnetic field the sum rule is violated by the NRG approach to a different extent in the positive and negative frequency ranges, which leads to jumps at $\omega=0$ in the spectral functions (see Fig. (4), while this problem is absent in the DM-NRG approach.

Spectral functions display universal scaling in the vicinity of $T_{h} \underline{\underline{26}}$ In Fig. 囵 we show how the spectral functions of the composite fermion operator, $F_{1}^{\dagger} \equiv f_{0,1}^{\dagger} \vec{S} \vec{\sigma}$ can be scaled on top of each other using the scale $T_{h}$. Although, this collapse can be obtained in both approaches, there is an $\approx 20 \%$ jump at $\omega=0$ in the NRG results while the DM-NRG results are continuous there. It is possible to eliminate the jump in the NRG results by determining the phase shifts from the energy spectrum

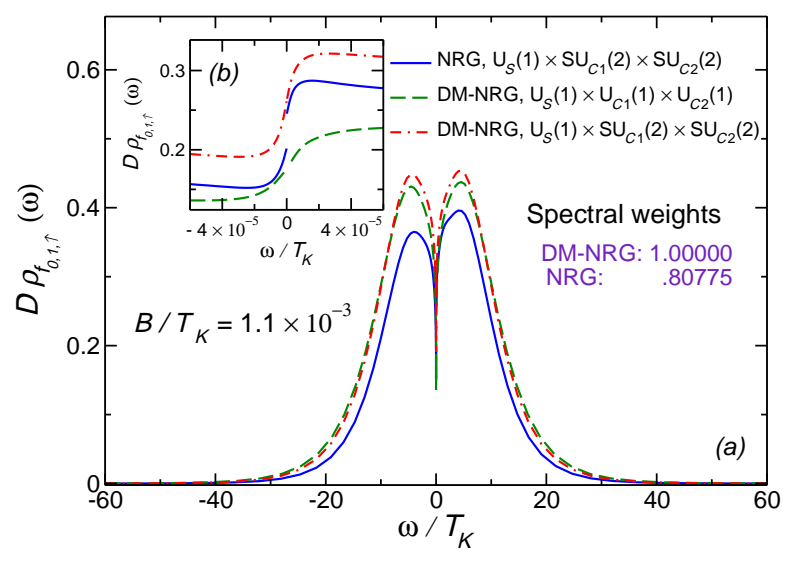

FIG. 4: (color online) Dimensionless spectral function of $f_{0,1, \uparrow}$ normalized by $D$, the bandwidth cut-off, as a function of $\omega / T_{K}$ in the presence of magnetic field obtained with DMNRG and with NRG using the symmetries: $\mathrm{U}_{S}(1) \times \mathrm{SU}_{C 1}(2) \times$ $\mathrm{SU}_{C 2}(2)$ and $\mathrm{U}_{S}(1) \times \mathrm{U}_{C 1}(1) \times \mathrm{U}_{C 2}(1)$. (b) On a smaller scale at $\omega=0$ we show the smoothness of the DM-NRG data using both groups and the jump in the NRG results using the larger group.

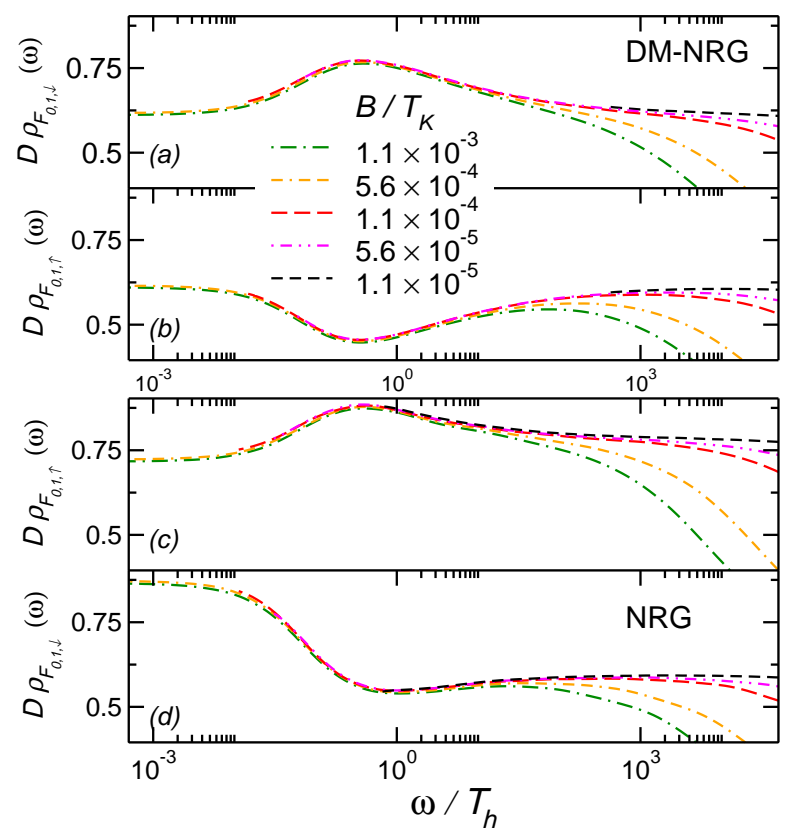

FIG. 5: (color online) Dimensionless spectral function of the $\downarrow-(a),(c)$ and the $\uparrow$-spin $(b),(d)$ components of the local composite fermion operator normalized by $D$, the bandwidth cutoff, for sufficiently small values of $B$ as a function of $\omega / T_{h}$ scaled on top of each other using NRG and DM-NRG together with the group $\mathrm{U}_{S}(1) \times \mathrm{SU}_{C 1}(2) \times \mathrm{SU}_{C 2}(2)$.

with high precision, but even after these corrections, the results continue to violate the sum rule and numerical errors for low frequencies remain of the same size as before. Moreover, the 'universal' curve obtained by conventional NRG is clearly incorrect and different from the DM-NRG result. Also, if we try to compute the imaginary part of 
the on-shell $T$-matrix of the $2 \mathrm{CK}$ model where the local composite fermion spectral functions for both $\uparrow$ - and $\downarrow$ spin components have to be summed up, we end up with large numerical errors in the NRG results (see Fig. 6), while DM-NRG provides satisfactory results even in this case. It is thus clear from these examples that the DMNRG method together with the use of lots of symmetries produces much more reliable results than NRG with non-Abelian symmetries or DM-NRG with only Abelian symmetries, and its use is needed to do computations for more delicate quantum impurity models.

\section{CONCLUSIONS}

To summarize, in this paper, we have shown how the recently developed spectral sum-conserving DM-NRG methods can be used in the presence of any number and type of non-Abelian symmetries. The most important result of this paper is a very general and simple recursion relation for the truncated reduced density matrix, which enables one to compute the spectral properties of any local correlation function in the presence of non-Abelian symmetries. The expressions derived hold for almost any symmetry, including non-Abelian finite groups, point groups, SU(N) groups, and, of course, Abelian groups such as $\mathrm{U}(1)$ or the parity. Using these symmetries, reduces considerably the time needed for the computations and enhances significantly the accuracy of the numerical results for dynamical quantities. The general formulation presented in this paper also allowed us to construct a flexible DM-NRG code where symmetries are handled

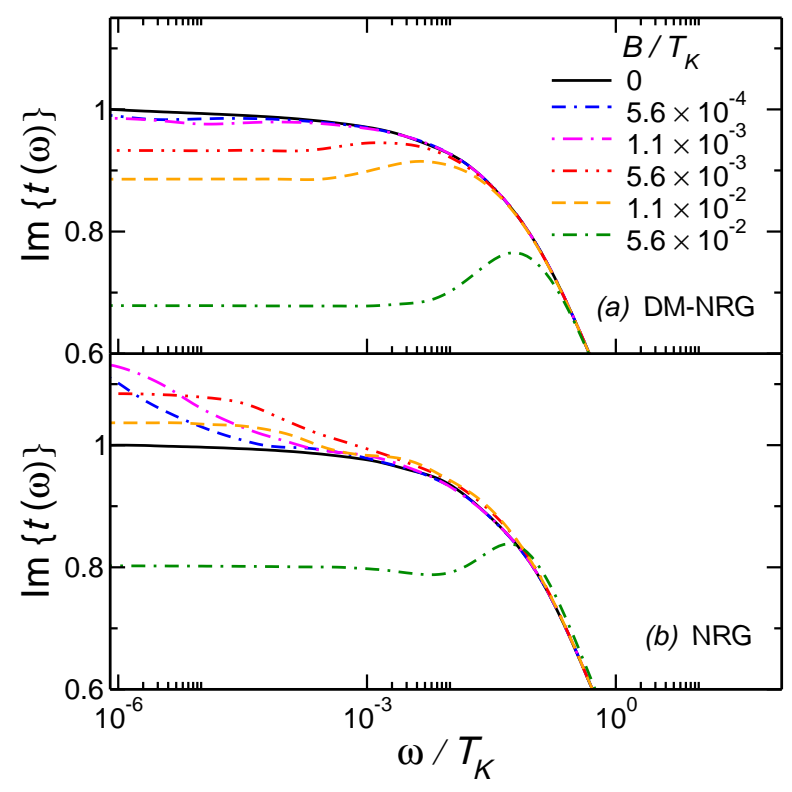

FIG. 6: (color online) Imaginary part of the on-shell $T$ matrix using DM-NRG and NRG together with the group $\mathrm{U}_{S}(1) \times \mathrm{SU}_{C 1}(2) \times \mathrm{SU}_{C 2}(2)$ for various magnetic field values as a function of $\omega / T_{K}$. dynamically, and which is able to learn and handle essentially any type of symmetry 31

We demonstrated the advantages of the generalized method by applying it to the two-channel Kondo model in a magnetic field. The presence of magnetic field makes this model very challenging from the point of view of NRG calculations. We have carried out calculations for the local fermion's and for the local composite fermion's spectral function at zero temperature and in a finite magnetic field. In conventional NRG, there is always a jump at $\omega \rightarrow 0$ between the positive and negative frequency parts of the spectral function. In addition to being spectral sum-conserving, the use of DM-NRG almost completely eliminated this jump and made it possible to compute the universal cross-over curves in a magnetic field. Moreover, the DM-NRG approach used with larger symmetry groups has provided substantially better results for the spectral functions: In the limit $\omega \rightarrow 0$ we needed to use non-Abelian symmetries in the DM-NRG approach to recover the expected power law behavior known from conformal field theory. Also, the universal crossover curve and the shape of the the peak at the renormalized magnetic field $T_{h} \propto B^{2} / T_{K}$ was much better resolved by DM-NRG than by NRG, and thus DM-NRG with nonAbelian symmetries lead to much more reliable results for the $T$-matrix (see Fig. 5) than NRG with non-Abelian or DM-NRG with Abelian symmetries. We emphasize that, to obtain the $\omega=0$ value of the spectral function correctly as well as its proper scaling behavior, we needed to use non-Abelian symmetries.

The method presented here thus opens up the possibility to carry out very accurate spectral sum-conserving DM-NRG calculations for multichannel systems, such as multi-dot devices, and to perform reliable DMNRG-DMFT calculations for multichannel lattice models. Combined with the matrix product state approach, it might also provide a way to use methods applied in DMRG to improve the high frequency resolution of NRG.

\section{Acknowledgments}

We are deeply indebted to Ireneusz Weymann, who participated in the initial stage of the construction of the flexible NRG code, and to László Borda for his help and many comments. We would like to thank Frithjof Anders, Jan von Delft and Andreas Weichselbaum for useful discussions. This research was supported partly by the Hungarian Research Fund (OTKA) under Grant Nos. NF 61726 and K 68340 and by the János Bolyai Research Fund. A.T. is grateful to the Institute of Mathematics of the BUTE for providing access to their computer cluster, supported by OTKA under grant No. 63066. CPM was partially supported by the Romanian Grant No. CNCSIS $780 / 2007$. 


\section{PROOF OF THE DIAGONAL FORM OF THE REDUCED DENSITY MATRIX FOR SU(2) SYMMETRIES}

In this appendix we prove, in a different way from how it was demonstrated in the main part of the article, the general theorem for the diagonal form of the reduced density matrix presented in Section III in the case when only $\mathrm{SU}(2)$ local symmetries are involved.

The proof for the diagonal form of the reduced density matrix goes by induction for the iteration steps. At the last iteration, by construction the reduced density matrix $R^{[N]}$ is scalar under the local symmetry group (cf. Eq. (39) ). Due to this invariance by very general considerations $R^{[N]}$ can be written as a sum over tensor products of irreducible tensor operator components, and is of the form

$$
R^{[N]}=\sum_{\alpha}\left(T_{\alpha}^{[l o c]}\right)_{\underline{t}, \underline{t}^{z}}^{\dagger} \otimes\left(T_{\alpha}^{[N-1]}\right)_{\underline{\underline{t}, \underline{t}^{z}}},
$$

where $\left(T_{\alpha}^{[l o c]}\right)_{\underline{t}, \underline{t}^{z}}$ and $\left(T_{\alpha}^{[N-1]}\right)_{\underline{t}, \underline{t}^{z}}$ are irreducible tensor operator components of the same rank $\underline{t}$ acting on the local vector space at site $N$ and on the rest of the Wilson chain, respectively and $\alpha$ labels all possible tensor operators. This special form is a consequence of the invariance of $R^{[N]}$ under the local symmetry transformations. 28

To obtain $R_{K K}^{[N-1]}$ we have to trace over the local basis states, $\left\{\left|\mu, \underline{q}_{\mu}, \underline{q}_{\mu}^{z}\right\rangle\right\}$, that is we have to compute the following matrix elements

$$
\begin{aligned}
& \left\langle u\left\|R_{K K}^{[N-1]}\right\| v\right\rangle=\sum_{\alpha, \underline{q}_{\mu}, \mu} \operatorname{sgn}\left(T_{\alpha}^{[N-1]}, \underline{q}_{\mu}\right) \\
& \times\left\langle u\left\|T_{\alpha}^{[N-1]}\right\| v\right\rangle\left\langle\mu\left\|T_{\alpha}^{[l o c]}\right\| \mu\right\rangle \\
& \times\left\langle\underline{Q}_{u} \underline{Q}_{u}^{z} \mid \underline{t}^{z} \underline{Q}_{v} \underline{Q}_{v}^{z}\right\rangle \\
& \times \sum_{\underline{q}_{\mu}^{z}=-\underline{q}_{\mu}}^{\underline{q}_{\mu}}\left\langle\underline{q}_{\mu} \underline{q}_{\mu}^{z} \mid \underline{t}^{z} \underline{q}_{\mu} \underline{q}_{\mu}^{z}\right\rangle .
\end{aligned}
$$

In Eq. (52) we have applied the Wigner-Eckart theorem. The sign factor $\operatorname{sgn}(.,$.$) depends on the number$ of fermionic operators used in the construction of $T_{\alpha}^{[N-1]}$ and the local states. In Eq. (52) in the last sum only the terms with $\underline{t}_{z}=0$ give non-vanishing contributions and the sum can be reduced to

$$
\sum_{q^{z}=-q}^{q}\left\langle q q^{z} \mid t 0 q q^{z}\right\rangle=(2 q+1) \delta_{t, 0} .
$$

Eq. (53) implies that the only non-vanishing contributions are those corresponding to $\underline{t}=\underline{t}^{z}=0$, i.e. to scalar $T_{\alpha}^{[l o c]}$ and $T_{\alpha}^{[N-1]}$. Therefore the reduced density matrix $R^{[N-1]}$ is diagonal in the representation indices. The induction towards smaller iteration steps goes recursively the same way.
${ }^{1}$ For a review, see e.g. L. I. Glazman, M. Pustilnik, in "Nanophysics: Coherence and Transport," eds. H. Bouchiat et al., pp. 427-478 (Elsevier, 2005).

2 A. Georges, G. Kotliar, W. Krauth and M. J. Rozenberg. Rev. Mod. Phys. 68, 13 (1996).

3 N. Andrei and C. Destri, Phys. Rev. Lett. 52, 364 (1984).

4 A. M. Tsvelick and P. B. Wiegmann, J. Stat. Phys. 38, 125 (1985).

${ }^{5}$ I. Affleck and A. W. W. Ludwig, Nucl. Phys. B 352, 849 (1991); ibid 360, 641 (1991), I. Affleck et al., Phys. Rev. B 45, 7918 (1991).

${ }^{6}$ V. J. Emery and S. Kivelson, Phys. Rev. B 47, 10812 (1992).

7 A. A. Abrikosov, Physics 2, 5 (1965).

8 M. Fowler and A. Zawadowski, Sol. St. Comm. 9, 471 (1971).

9 Ph. Nozières, J. Low Temp. Phys. 17, 31 (1974).

10 K. G. Wilson, Rev. Mod. Phys. 47, 773 (1975); for a recent review of NRG see R. Bulla, T. Costi, T. Pruschke, arXiv:cond-mat/0701105.

11 L. N. Oliveira and J. W. Wilkins, Phys. Rev. B 244863 (1981), ibid 32696 (1986).

12 T. A. Costi and A. C. Hewson, Phil. Mag. B 651165 (1992).

13 T. A. Costi, A. C. Hewson and V. Zlatić, J. Phys. Cond. Mat. 62519 (1994).
14 S. Suzuki, O. Sakai and Y. Shimizu, J. Phys. Soc. Jpn. 65 4034 (1996).

15 W. Hofstetter, Phys. Rev. Lett. 85, 1508 (2000)

16 F. B. Anders and A. Schiller, Phys. Rev. Lett. 95, 196801 (2005)

17 R. Peters, T. Pruschke, F. B. Anders, Phys. Rev. B 74, 245114 (2006) arXiv:cond-mat/0607494.

18 A. Weichselbaum, J. von Delft, Phys. Rev. Lett. 99, 076402 (2007) arXiv:cond-mat/0607497.

19 R. Zitko, J. Bonca, Phys. Rev. B 73, 035332 (2006).

20 D. L. Cox and A. Zawadowski, Adv. Phys. 47, 599 (1998).

${ }^{21}$ G. Zaránd et al. (unpublished).

22 R. M. Potok, I. G. Rau, H. Shtrikman, Y. Oreg and D. Goldhaber-Gordon, Nature (London) 446, 167 (2007).

23 B. A. Jones, C. M. Varma and J. W. Wilkins, Phys. Rev. Lett. 61, 125 (1987).

24 J. F. Cornwell:, Group Theory in Physics, An Introduction, Academic Press (1997).

25 A. I. Tóth, L. Borda, J. von Delft and G. Zaránd, Phys. Rev. B 76, 155318 (2007).

26 A. I. Tóth and G. Zaránd, arXiv:0801.4272.

27 K. Le Hur, Phys. Rev. Lett. 92, 196804 (2004).

28 A. I. Tóth, Ph. D. Thesis (unpublished).

29 The eigenvalues of the operators $\vec{S}^{2}$ and $\vec{C}^{2}$ are given by $S(S+1), C(C+1)$.

30 Some of the considerations presented here do not carry 
over for non-compact Lie Groups.

31 This code has open access and, together with a detailed description, it will be accessible soon at the site http://www.phy.bme.hu/ ${ }^{\sim}$ dmnrg. 